\title{
ELECTROCHROMIC DISPLAYS
}

\author{
R. HURDITCH \\ The Allen Clark, Research Centre, Plessey Co. Ltd., Caswell, Northants, England.
}

(Received January 27, 1977)

\section{INTRODUCTION}

A number of electro-optic, electrolytic and other effects can give rise to reversible changes in the absorbance or reflectivity of solids and liquids under the application of an external field. Such effects may be grouped under the general heading of "electrochromism" when the spectral changes are induced by an electric field or current and occur in the visible part of the spectrum. Any electrochromic effect in which there is a significant change in transmission offers potential for applications involving the modulation of light. Electrolytic effects can involve large changes in transmission over a wide spectral bandwidth, and have been proposed for a variety of display applications in which they may compete with, for example, liquid crystals and electrophoretic display technologies ${ }^{1}$.

In its simplest form an electrolytic-electrochromic display device, illustrated schematically in Figure 1, is a reversible cell in which one electrode, called the active electrode, is optically isolated from the second, counter electrode, for example, by means of a porous reflective spacer, but can be viewed externally. An electrolyte, containing ions or molecules selected to undergo an electrocolouration reaction, fills the space between the electrodes. Under the application of a suitable external voltage, exchange reactions take place in which the active ions capture electrons at the cathode and release electrons at the anode, undergoing reduction or oxidation respectively, involving a visible absorbance change.

Although many reversible electrochemical reactions involve colour changes, only a few types are really suitable for display applications, since the coloured species are generally soluble ions which diffuse away from the active electrode leading to relatively poor contrast changes and rapid loss of colour when the driving voltage is removed. This problem is overcome if the coloured state is actually precipitated from the solution, i.e. deposited onto the active electrode; an analogous process to the "storage" mechanism of the lead-acid battery in which insoluble lead oxide is formed on the anode during charging. Thus, perhaps the simplest example of an electrochromic reaction which has been proposed for display applications is the reversible electroplating of silver from a solution containing silver salts, onto a transparent conducting cathode. ${ }^{2}$ A more complex example which has been developed by Philips and $\mathrm{ICI}^{3}$ involves the use of a redox-dye called a 'viologen' (a substituted 4-4'. bipyridinium compound) the structure of which has been modified so that the reduced coloured form, in the presence of a suitable anion (e.g. bromide ions), forms an insoluble salt which deposits onto the cathode as the electrode reduction reaction proceeds.

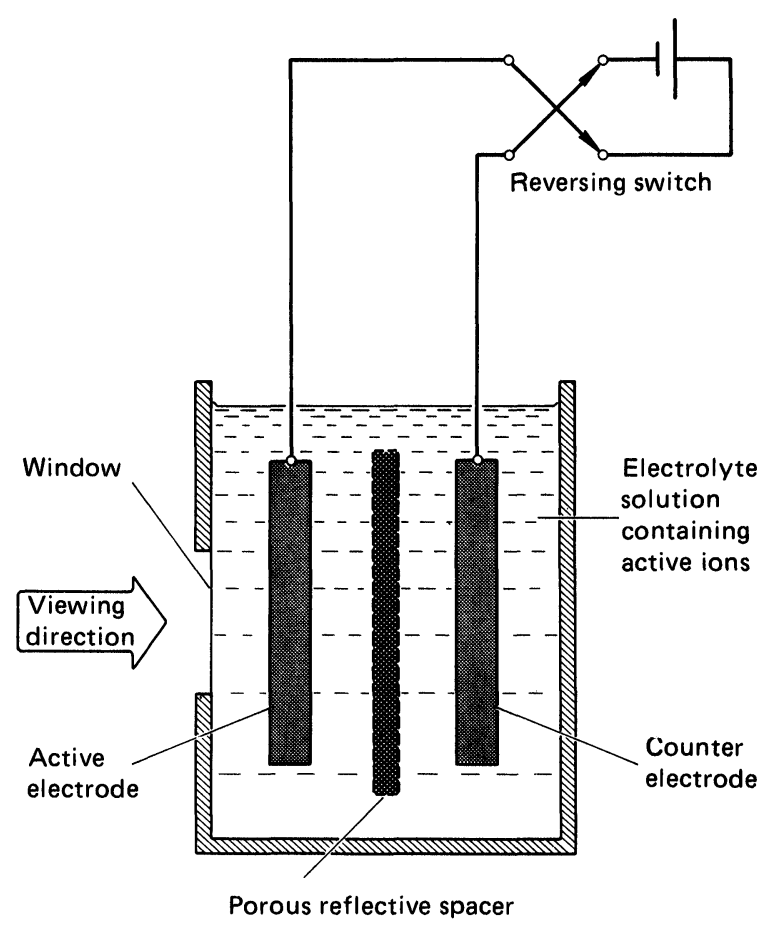

FIGURE 1 Schematic diagram of an electrochromic cell. 
For these electrochromic cells, the optical density (absorbance) changes are proportional to the thickness of the precipitated layer and hence to the number of molecules or ions converted during the electrode reaction. From Faraday's Laws it can be shown that a charge density of about $5 \mathrm{mC} / \mathrm{cm}^{2}$ is required to produce a corresponding optical density of 0.5 (corresponding to a maximum contrast of 10:1 for a reflective cell), following the one electron reduction or oxidation of a material to a product with an absorption coeffcient of $5 \times 10^{4} \mathrm{~cm}^{-1}$. Since most electrode processes taking place in solution can only be operated reversibly at low voltages over narrow voltage ranges and at current densities of less than a few hundred $\mathrm{mA} / \mathrm{cm}^{2}$, electrochromic displays are relatively slow $(10-500 \mathrm{msec})$ and consume approximately $10 \mathrm{~mJ} / \mathrm{cm}^{2}$ during switching.

The third important example in which persistence of the coloured state is achieved, involves the reduction and oxidation of a transition metal oxide film such as tungstic oxide in contact with a suitable electrolyte. In this case, the electrocolouration reaction occurs in a stationary phase previously deposited onto a conducting electrode. Electrochromic effects in transition metal oxide films have been studied by RCA, IBM, Zenith and others ${ }^{4}$ and are of particular interest because of the potential for producing a 'solid-state' electrochomic device which can be fabricated by standard thin film technology.
This aim has not yet been achieved, but neither has a complete understanding of the electrochromic mechanism. The results of our recent investigations of the electrochromic behaviour of tungsten oxide films are summarised in the following section.

\section{ELECTROCHROMISM IN EVAPORATED TUNGSTEN OXIDE FILMS}

The electrochromic behaviour of evaporated tungstic oxide $\left(\mathrm{WO}_{3}\right)$ films is most conveniently studied using samples prepared by evaporating $0.5-1.5 \mu \mathrm{m}$ thick films of $\mathrm{WO}_{3}$ onto transparent conducting indiumtin oxide (ITO)-coated glass substrates at substrate temperatures in the range $80-300^{\circ} \mathrm{C}$. Prepared in this manner, the $\mathrm{WO}_{3}$ films are amorphous. The ITO layer in contact with the $\mathrm{WO}_{3}$ film is made the active electrode of an electrolytic cell as shown in Figure 1, using dilute $\mathrm{H}_{2} \mathrm{SO}_{4}$ as electrolyte and a platinum gauze counter electrode. The application of negative potential greater in magnitude than $-0.7 \mathrm{~V}$ to the ITO- $\mathrm{WO}_{3}$ electrode causes the $\mathrm{WO}_{3}$ film to turn deep blue within $0.5-1.0$ seconds. The spectrum before and after colouration is shown in Figure 2. The blue state remains fixed with the voltage removed, but the initial transparent state of the $\mathrm{WO}_{3}$ film can be restored by applying a reverse, $+0.8 \mathrm{~V}$ potential to the ITO- $\mathrm{WO}_{3}$ electrode. Under controlled conditions

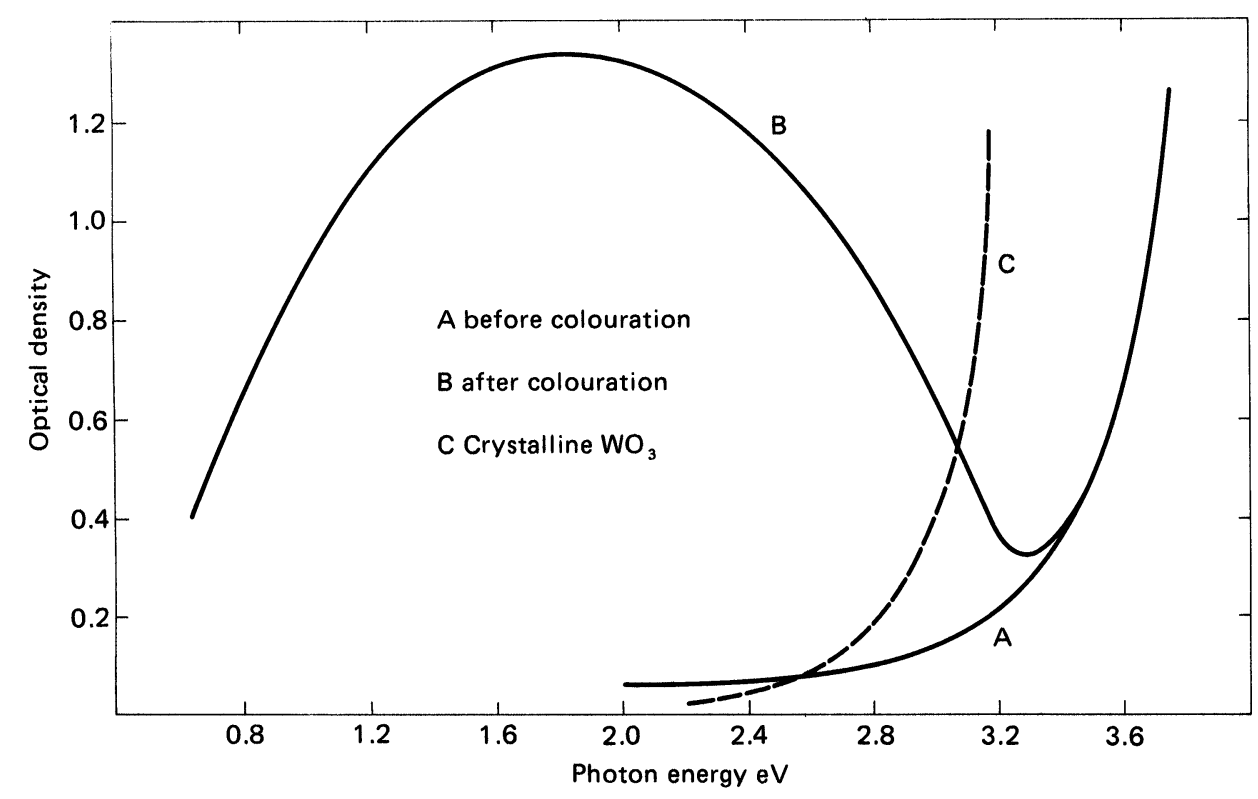

FIGURE 2 Transmission vs. photon energy for a $1.0 \mu \mathrm{m}$ electro-chromic $\mathrm{WO}_{3}$ film. 
the cycle of colouring and bleaching can be repeated more than $10^{6}$ times without significant degradation.

It has been established that the observed colour change is due to the formation of an amorphous compound, which can be formulated as $\mathrm{H}_{x} \mathrm{WO}_{3}$, by cathodic reduction involving the diffusion of electrons from the ITO cathode and $\mathrm{H}^{+}$ions from the electrolyte into the $\mathrm{WO}_{3}$ layer i.e.

$$
x e+x \mathrm{H}^{+}+\underset{\text { (Colourless) }}{\mathrm{WO}_{3}} \rightleftarrows \underset{\text { (blue) }}{\mathrm{H}_{x} \mathrm{WO}_{3}}
$$

The value of $x$ may vary from 0 to 0.36 , and the reaction occurs throughout the whole volume of the $\mathrm{WO}_{3}$ films.

Crystalline blue compounds of analogous composition called "hydrogen tungsten bronzes" can also be formed by reduction of tungstic oxide electrolytically, chemically (e.g. zinc + hydrochloric acid) or with gasphase hydrogen atoms. Many other related tungsten bronzes $\mathrm{M}_{x} \mathrm{WO}_{3}$ have been prepared ${ }^{5}$ where $\mathrm{M}$ may be one of a large number of metallic elements and $x$ can vary for any given element over the range from 0 to 1 . The ease with which the tungsten bronzes form, and their wide range of stoichiometry derives from the parent "Perovskite" structure of $\mathrm{WO}_{3}$ shown in Figure 3A, in which the sites between the octahedra can readily accommodate metal ions without serious distortion of the $\mathrm{WO}_{3}$ matrix. For the

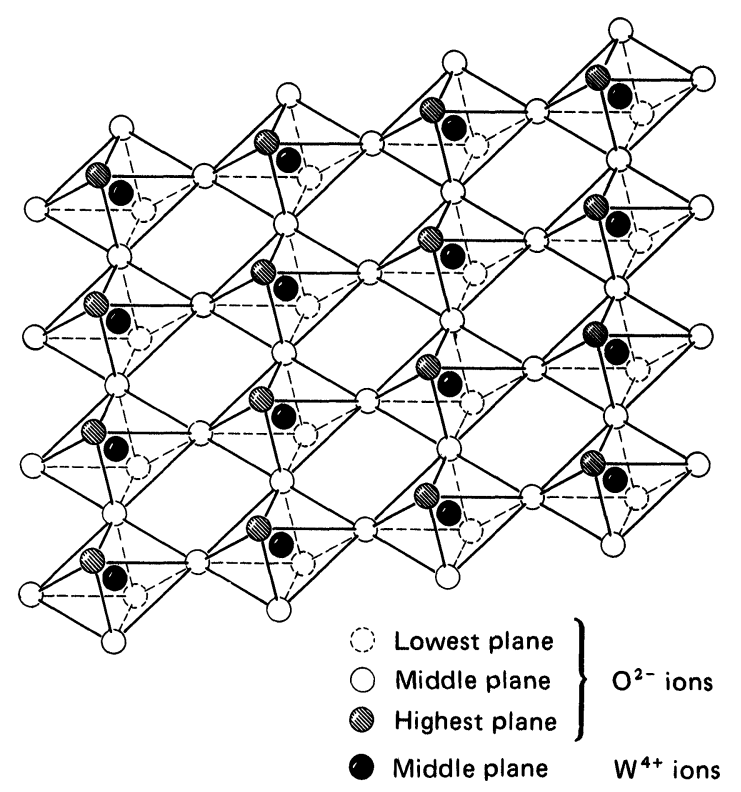

FIGURE 3A A plane of $\mathrm{WO}_{3}$ octahedra from the crystal lattice. case of $\mathrm{Na}_{\mathbf{x}} \mathrm{WO}_{3}$, in which the radius of $\mathrm{Na}^{+}(0.95 \AA)$ is almost identical to the radius of the ' $A$ '-site for the undistorted cubic $\mathrm{WO}_{3}$ matrix $(0.96 \AA)$, the maximum occupancy, $x=1$ may be achieved as shown in Figure 3B.

The most outstanding property of the tungsten bronzes, which is of particular importance in understanding the electrochromic properties of evaporated $\mathrm{WO}_{3}$ films, is that the electrons (i.e. those required to preserve neutrality) associated with the added ions $\mathrm{M}^{\mathrm{n}+}$, whatever these ions may be, are essentially free to conduct for all values of $\mathrm{x}$. Typical metallic conductivities of $10^{3}-10^{4}(\Omega-\mathrm{cm})^{-1}$ are obtained when the product $\mathrm{nx}$ exceeds 0.25 . This property stems from the relatively large overlap between atomic orbitals on $\mathrm{W}^{6+}$ and $\mathrm{O}^{2-}$ ions and leads to a lower energy for an electron in the resulting conduction band compared with that of an electron in an atomic orbital on $\mathrm{W}^{6+}$ (i.e. $\mathrm{W}^{5+}$ ).

Relatively high conductivities of the order $1-100$ $(\Omega-\mathrm{cm})^{-1}$ have also been measured for the blue phase of electro-coloured $\mathrm{WO}_{3}$ films compared with values of $10^{-4}-10^{-6}(\Omega-\mathrm{cm})^{-1}$ for the uncoloured state. A "free" electron concentration of approx. $10^{21} \mathrm{~cm}^{-3}$ is required to account for the high conductivity in the coloured state.

The origin of the very broad visible-infrared absorbance of the blue phase (Figure 2) may be due to the presence of the relatively high concentration of "free" electrons. For the case of the crystalline sodium tungsten bronzes it has been proposed that the colours, which change from blue, through purple, red, orange and yellow, as $x$ increases from 0.2 to

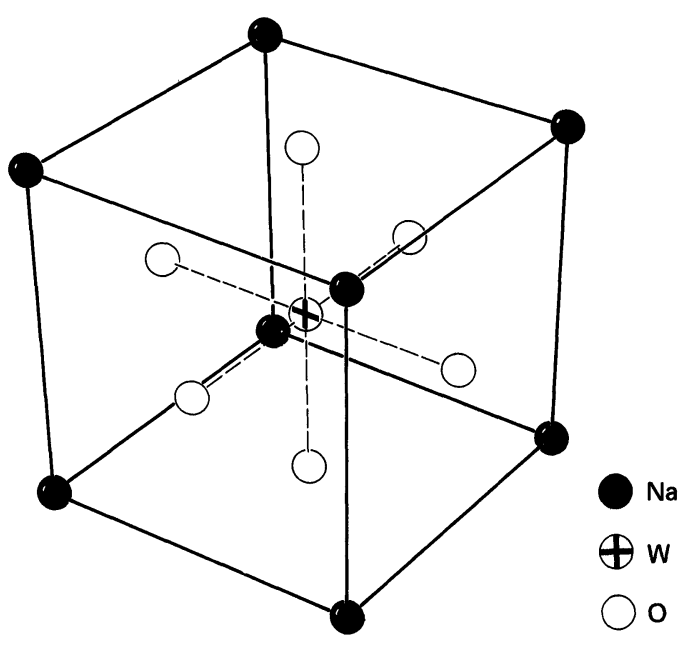

FIGURE 3B Perovskite structure of $\mathrm{NaWO}_{3}$. 
0.9 , are due to plasma resonance of the free electrons. In simple metals, plasma resonance gives rise to a reflectivity minimum in the ultraviolet which increases in energy with increase in electron concentration. In the tungsten-bronzes, this is red-shifted because of interactions with the highly polarisable electrons associated with $\mathrm{O}^{2-}$ ions.

The kinetics of the electro-colouring and bleaching processes can be studied by measuring the time depen. dence of current density $j$, and transmission as a function of $\mathrm{WO}_{3}$ film thickness and electrolyte concentration. It is found that the induced optical density change is proportional to the charge density, $\int j d t$, over a large range of change in transmission (as shown in Figure 4). This result confirms that Faraday's Laws are obeyed in this system. It has also been found that the diffusion of $\mathrm{H}^{+}$ions at the $\mathrm{WO}_{3}$-electrolyte interface and within the $\mathrm{WO}_{3}$ layer determines the rate of colouring. However, the estimated value of the diffusion coefficient for $\mathrm{H}^{+}$ions in the solid film of $10^{-8}$ to $10^{-6} \mathrm{~cm}^{2} / \mathrm{sec}$, depending upon the prepa. ration conditions of the evaporated film, is much greater than values calculated from nuclear magnetic resonance (N.M.R.) studies of proton relaxation in crystalline hydrogen bronzes. $\left(10^{-9}-10^{-11} \mathrm{~cm}^{2} / \mathrm{sec}\right)$. This important difference may be attributed to the "porous" nature of the amorphous films, which freely absorb water, the presence of which promotes more rapid diffusion of protons than in the "nonporous" crystalline material. Further studies are being undertaken in order to gain a more complete and quantitative understanding of the colouring and bleaching processes.

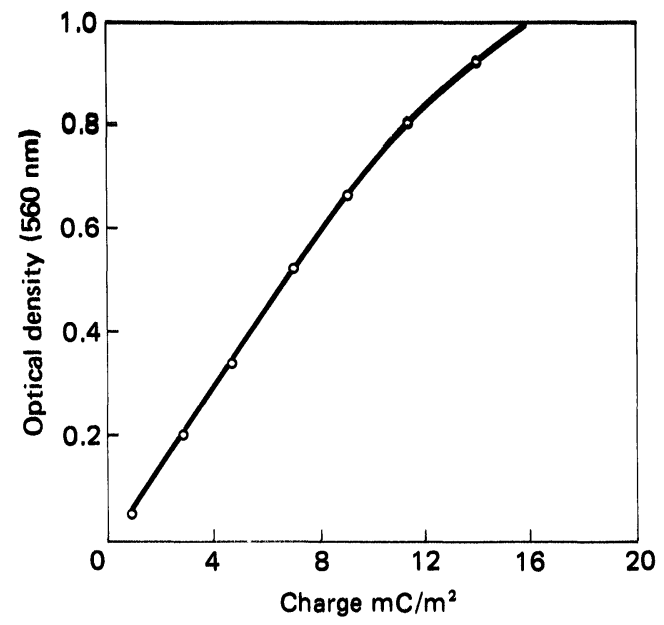

FIGURE 4 Optical density vs. charge density for an electrochromic cell.

\section{DISPLAY DEVICES AND APPLICATIONS}

A typical single aperture display device incorporating several features designed to optimise the operational lifetime is shown in Figure 5. The platinum counterelectrode of the experimental system has been replaced by a $\mathrm{WO}_{3}-\mathrm{Au}$ electrode which eliminates electrolysis of water and the formation of gas bubbles.

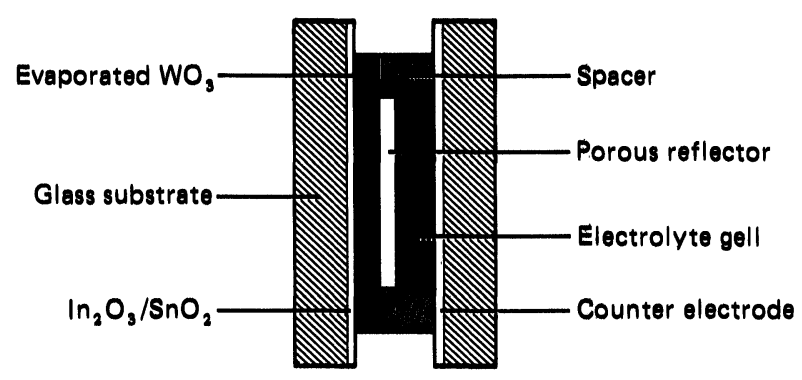

FIGURE 5 Schematic diagram of reflective electrochromic cell.

This occurs to a small extent when a metal or graphite counter electrode is employed, since the required cell voltage is then close to the decomposition potential of water. 3M Sulphuric acid is gelled with polyvinyl alcohol and glycerol to form a semi-solid high conductivity electrolyte which does not require a vacuum tight seal. A porous reflective filter which may be dyed to give various colour contrasts (blue vs. yellow, red etc.) is placed in contact with the active electrode. Such a device can be operated up to $10^{6}$ cycles with switching times of $0.4 \mathrm{sec}$ without significant deterioration of performance. However, the shelf life, which may be as low as a few weeks, is found to be strongly dependent upon the quality of the sputtered ITO layer. This must be free from dust and particles in the micron size range, the presence of which may give rise to high-current density spots, which cause pinholes to form in the $\mathrm{WO}_{3}$ layer, leading to electrolytic reduction and gradual dissolution of the ITO layer beneath.

Similar devices have been constructed, notably by Sharp ${ }^{6}$ and Zenith, in which $\mathrm{LiClO}_{4}$ dissolved in a non-aqueous solvent replaces $\mathrm{H}_{2} \mathrm{SO}_{4}$, and the electrochromic reaction involves the formation of a lithium tungsten bronze

$$
x \mathrm{Li}^{+}+x e+\mathrm{WO}_{3} \rightleftharpoons \mathrm{Li}_{x} \mathrm{WO}_{3} .
$$

These devices are slower owing to the lower rates of diffusion of $\mathrm{Li}^{+}$ions compared with $\mathrm{H}^{+}$ions in the evaporated $\mathrm{WO}_{3}$ layer. 
Although electrochromic devices are relatively slow, with present minimum switching times of $50 \mathrm{msec}$ for devices based on the viologens, they have the potential advantages of low cost, ease of large area fabrication and zero power consumption when not being updated. Further materials developments are feasible in both the organic and metal-oxide systems, which may lead to improvements over present performance and lifetimes. The most important applications, apart from digital watches, could be as an alternative to electromagnetic devices for large format alpha numeric displays, or for simple "on-off" indicators.

\section{REFERENCES}

1. A. R. Kmetz, F. K. Willisen (eds.), Non Emissive Electrooptic Displays, Plenum Press, 1976.

2. A. S. Hoffman, J. Opt. Soc. Am. 56 (6), 828 (1966).

3. C. J. Shoot et al., Appl. Phys. Lett., 23 (2), 64-5 (1973).

4. Society for Information and Display, Symposium, Washington, D.C., April 22, 1975, Session IV, Lewis Winner N.Y. 1975 .

5. P. G. Dickens, M. S. Whittingham, Quat. Rev., 22 (1) 30-44 (1968).

6. H. N. Hersh et al., Appl. Phys. Lett., 27 (12) 646-8 (1975). 

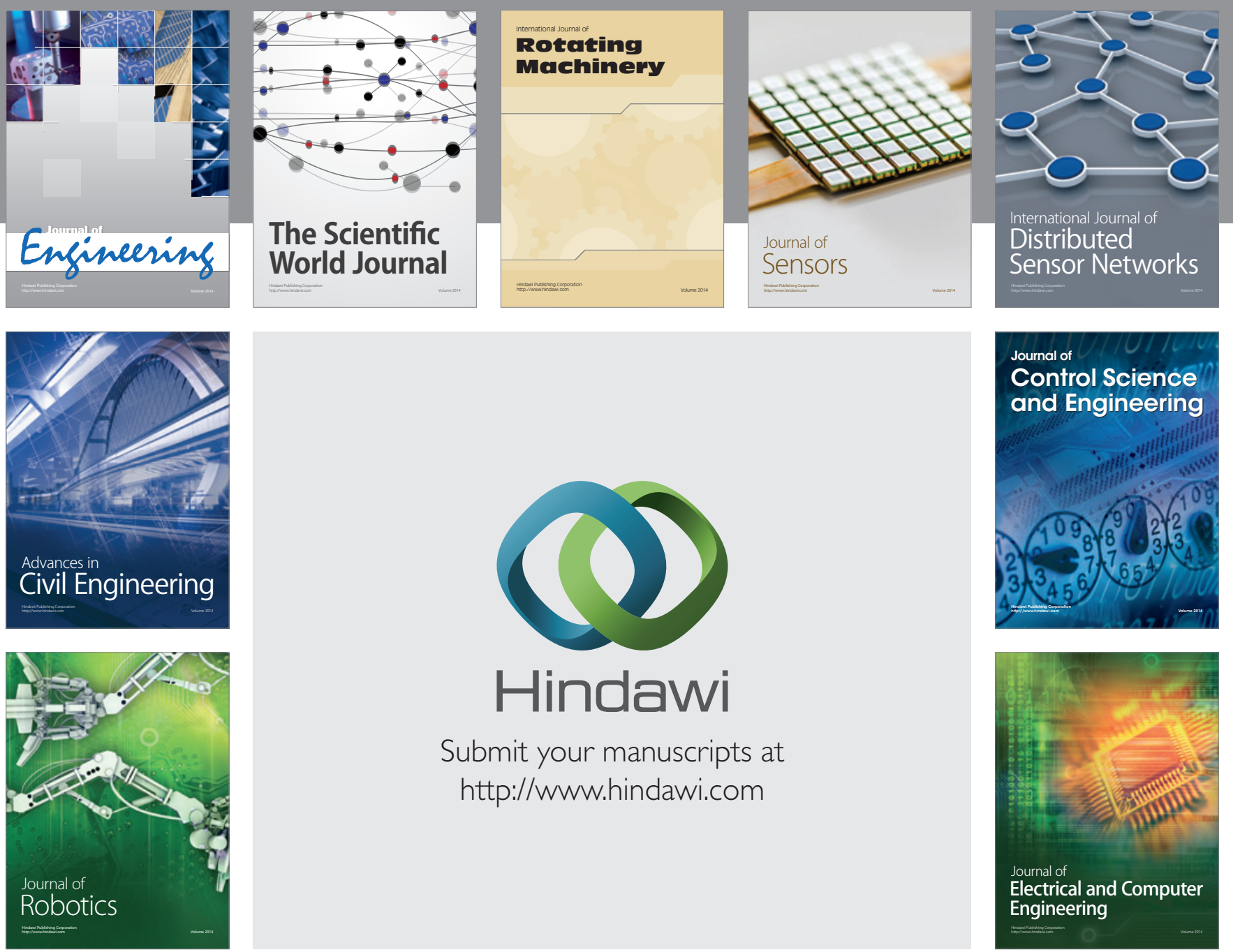

Submit your manuscripts at

http://www.hindawi.com
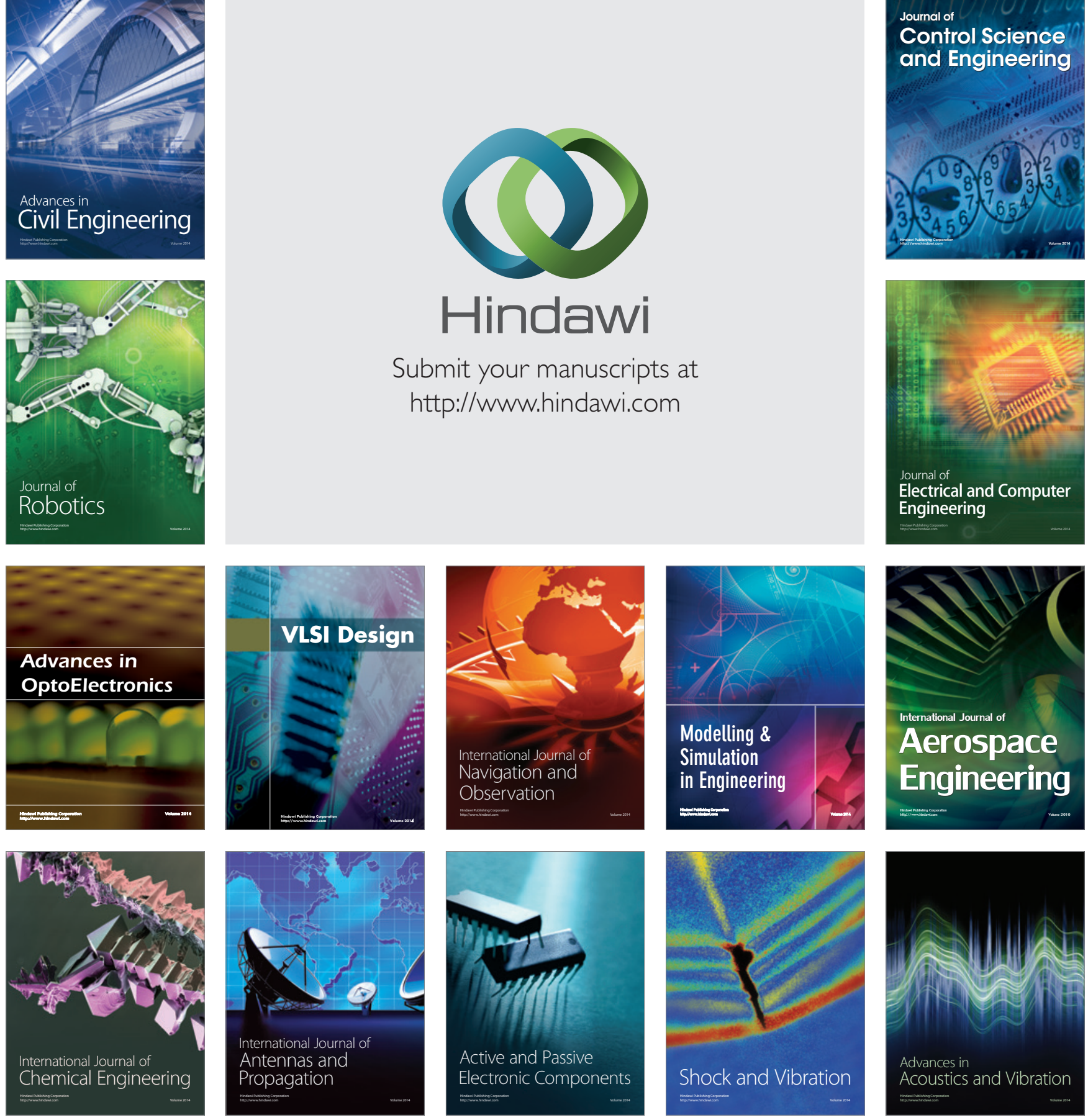